\title{
Goldmann tonometer error correcting prism: clinical evaluation
}

This article was published in the following Dove Press journal:

Clinical Ophthalmology

3 May 2017

Number of times this article has been viewed

\section{Sean McCafferty ${ }^{1-3}$ \\ Garrett Lim² \\ William Duncan ${ }^{2}$ \\ Eniko T Enikov ${ }^{4}$ \\ Jim Schwiegerling' \\ Jason Levine ${ }^{1,3}$ \\ Corin $\mathrm{Kew}^{3}$}

'Department of Ophthalmology,

College of Optical Science, University

of Arizona, ${ }^{2}$ Intuor Technologies,

${ }^{3}$ Arizona Eye Consultants,

${ }^{4}$ Department of Aerospace and

Mechanical, College of Engineering,

University of Arizona, Tucson, AZ, USA

Video abstract

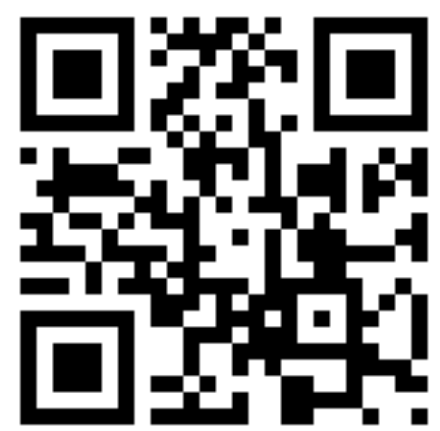

Point your SmartPhone at the code above. If you have a QR code reader the video abstract will appear. Or use:

http://youtu.be/qppbjAssyoy

Correspondence: Sean McCafferty Arizona Eye Consultants, LLC 6422 E. Speedway Blvd, Tucson, Suite 100, AZ 857I0, USA

Tel +I 5203273487

Email sjmccafferty66@hotmail.com
Purpose: Clinically evaluate a modified applanating surface Goldmann tonometer prism designed to substantially negate errors due to patient variability in biomechanics.

Methods: A modified Goldmann prism with a correcting applanation tonometry surface (CATS) was mathematically optimized to minimize the intraocular pressure (IOP) measurement error due to patient variability in corneal thickness, stiffness, curvature, and tear film adhesion force. A comparative clinical study of 109 eyes measured IOP with CATS and Goldmann prisms. The IOP measurement differences between the CATS and Goldmann prisms were correlated to corneal thickness, hysteresis, and curvature.

Results: The CATS tonometer prism in correcting for Goldmann central corneal thickness (CCT) error demonstrated a reduction to $< \pm 2 \mathrm{mmHg}$ in $97 \%$ of a standard CCT population. This compares to only $54 \%$ with CCT error $< \pm 2 \mathrm{mmHg}$ using the Goldmann prism. Equal reductions of $\sim 50 \%$ in errors due to corneal rigidity and curvature were also demonstrated.

Conclusion: The results validate the CATS prism's improved accuracy and expected reduced sensitivity to Goldmann errors without IOP bias as predicted by mathematical modeling. The CATS replacement for the Goldmann prism does not change Goldmann measurement technique or interpretation.

Keywords: glaucoma, tonometry, Goldmann, IOP, intraocular pressure, appalnation tonometer, corneal biomechanics, CATS tonometer, CCT, central corneal thickness, tonometer error

\section{Introduction}

Goldmann applanation tonometry (GAT) remains the standard of measurement for intraocular pressure (IOP). ${ }^{1-5}$ Numerous significant errors in GAT IOP measurements were identified mostly related to patient variability in corneal thickness, rigidity, curvature, and corneal tear film adhesion. ${ }^{6-8}$ Applanation tonometry theory assumes the cornea to be an infinitely thin membrane requiring no force to applanate except that produced by the IOP. Much of the GAT IOP measurement error is due to the inaccuracy of this assumption. ${ }^{5,7}$ GAT errors are significantly affected by the geometric properties of corneal thickness and corneal curvature. Variable material properties of the cornea such as Young's and shear moduli of elasticity both significantly affect the applanating force of the cornea adding to GAT error. ${ }^{9-11}$ An adhesion force created by the surface tension in the tear film also adds some highly variable error. ${ }^{12,13}$ However, no clinical quantification of IOP error due to this adhesive capillary force has been demonstrated.

The problems associated with GAT errors were highlighted by the Ocular Hypertension Treatment Study (OHTS), noting that pressure readings tend to be overestimated in thick and underestimated in thin corneas leading to a misdiagnosis of glaucoma. ${ }^{14,15}$ Central corneal thickness (CCT) correction is an incomplete correction for total GAT error and its use without other corrections has questionable 
utility. ${ }^{16}$ The effects of laser-assisted in situ keratomileusis (LASIK) surgery have been shown to render GAT IOP measurements, inaccurate. ${ }^{15}$ The practice of utilizing complex multiparameter algorithms to correct the GAT measurement errors and yield a more accurate IOP is cumbersome, leading to minimal clinical adoption with the exception of CCT. ${ }^{17}$

The CATS tonometer prism is a modification of the GAT which optimizes the corneal applanating surface of the flat GAT prism. The CATS prism is an investigational device and has not been approved for clinical use. Clinical application of the CATS prism including the force to pressure conversion was designed to be unchanged from the GAT prism. A modified Goldmann or correcting applanation tonometry surface (CATS) prism design was optimized to minimize the IOP measurement error due to corneal thickness, stiffness, curvature, and tear film adhesion. Mathematical modeling with finite element analysis and manometric IOP referenced cadaver eyes were used to optimize and validate the design. The optimized CATS prism was designed to measure the same pressure as a GAT prism in corneas with measured average corneal properties in corneal thickness, curvature, rigidity, and tear film. ${ }^{18}$ However, $\sim 50 \%$ of the patient population do not have average corneas. ${ }^{6,8,14,15}$

This clinical study was designed to evaluate a modified applanation prism surface demonstrating decreased sensitivity to all identified GAT errors and verify previously published mathematical modeling. ${ }^{18}$

\section{Methods}

The CATS tonometer prism, illustrated in Figures 1 and 2, mathematically reduces the GAT IOP measurement error due to all recognized variations in corneal biomechanics by $\sim 50 \%{ }^{18}$ All physical dimensions, materials, and properties of the CATS prism are identical to the GAT prism with the exception of the curved applanating surface of the prism. The CATS prism uses existing GAT or Perkins measurement armatures and the same practitioner protocol, including measurement technique, without calculations, or increased clinic time.

This clinical study was designed to examine the difference in IOP between the CATS prism and the GAT prism for a given subject. The IOP difference in the CATS and GAT measurements was correlated across all subjects to each of the corneal biomechanical errors. Observed correlations in the difference between the CATS and GAT were compared to published correlations between IOP error and each of the corneal biomechanical parameters. By convention, an increased negative slope in CATS-GAT difference

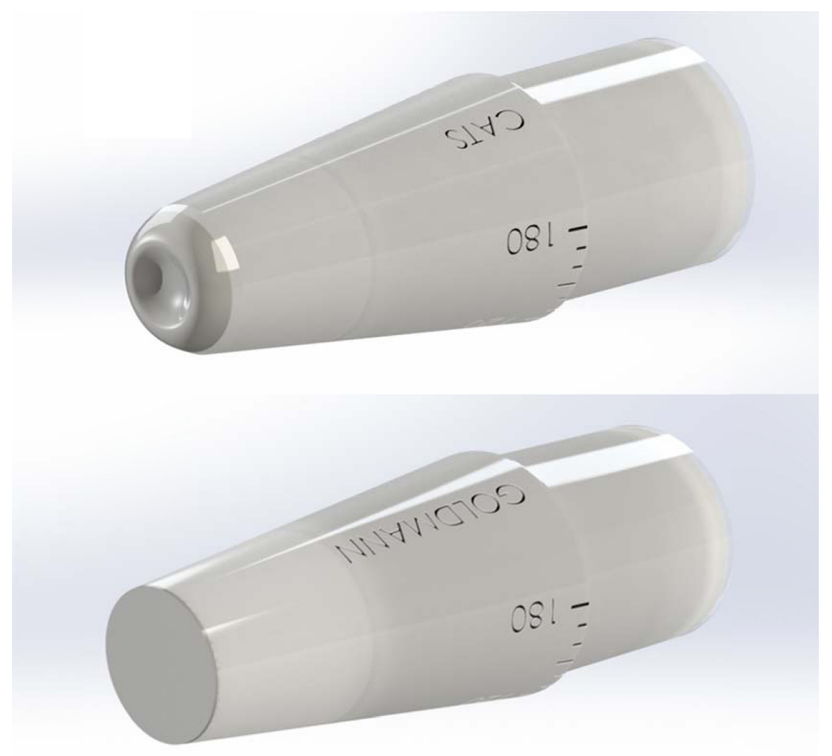

Figure I CATS versus GAT tonometer prism applanating surface.

Abbreviations: CATS, correcting applanation tonometry surface; GAT, Goldmann applanation tonometry.

correlation indicated a reduction in sensitivity to that error in the CATS prism.

The prospective clinical study was performed at two Arizona Eye Consultants offices in Tucson, AZ, USA. The study was conducted in accordance with the Declaration of Helsinki. All subjects gave written informed consent prior to participating in the study. Assessment by an IRB was completed on the clinical study use of the CATS prism and its use was deemed to be a negligible risk. Only the GAT IOP measurements were used to guide medical therapy.

One hundred nine eyes ( 55 patients) aged $\geq 18$ were enrolled from the clinic. A 100 eye sample size was sufficient to demonstrate statistically significant correlation

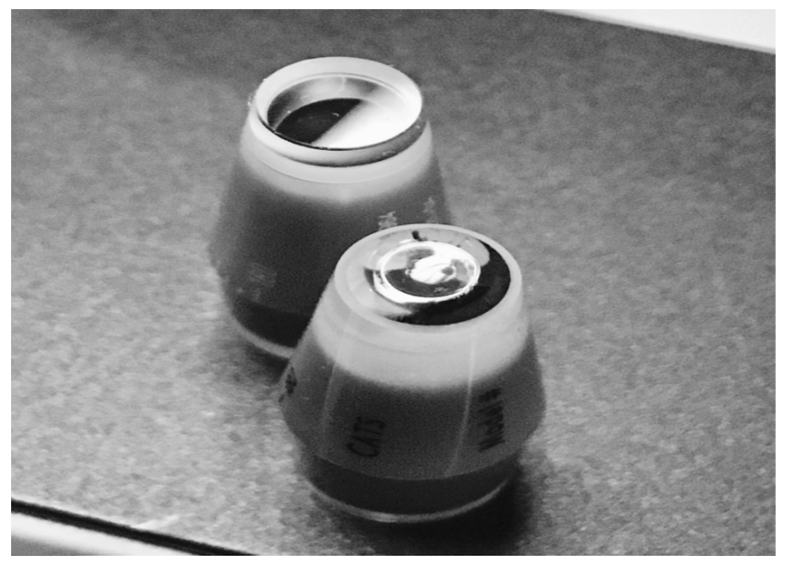

Figure 2 Photograph of the CATS tonometer prism applanating surface. Abbreviation: CATS, correcting applanation tonometry surface. 
from previous testing. Subjects were chosen from patients presenting for a routine examination requiring Goldmann tonometry. Included were subjects with binocular vision, able to fixate, and with IOP ranges from 5 to $35 \mathrm{mmHg}$. Subjects were selected in accordance with the following exclusion criteria: ocular surgery within the last 3 months; pregnant or nursing; only one functional eye; poor or eccentric fixation; high corneal astigmatism ( $>4.5$ diopters); corneal scarring; microphthalmos; buphthalmos; severe dry eyes; blepharospasm; nystagmus; keratoconus; or any other corneal or conjunctival pathology or infection.

Each subject underwent a standard ophthalmic exam by one of four trained and licensed investigators. An assistant investigator used an ocular response analyzer (ORA) (Reichert Ophthalmic Instruments, Depew, New York, NY) to measure the corneal resistance factor $(\mathrm{CRF})$ as an indicator of corneal biomechanical rigidity. A Zeiss HD-OCT-5000 spectral domain ocular coherence tomographer (Zeiss, Jena, Germany) was used by the assistant to measure CCT. Finally, the assistant investigator completed a corneal topography with a Zeiss Atlas model 9000 (Jena, Germany) and an averaged corneal curvature was used for analysis over the central $3 \mathrm{~mm}$ diameter of the cornea in accordance with ANSI Z80.23. Each investigator conducting IOP measurements was masked to the results of the assistant investigator's tests. Investigators were also masked to the randomized and alternated use of the CATS and GAT prism. Topical anesthetic drops with fluorescein (Fluorescein Sodium and Benoxinate Hydrochloride Ophthalmic Solution $0.25 \% / 0.4 \%$, Bausch and Lomb, Tampa, FL, USA) were applied prior to each measurement so that examination conditions were equivalent. Measurements of IOP were made two times with the CATS prism (one measurement was considered by averaging orthogonal measurements at $180^{\circ}$ and $90^{\circ}$ to correct for astigmatism) and two times with the Goldmann prism (again axis averaged). If the sequential measurements with one prism were $>2 \mathrm{mmHg}$ different, then a third measurement was obtained. All three measurements were then averaged. The third measurement was included in the study if it was within the range of the first two, otherwise all measurements were discarded. Four measurements were taken ( 2 with each prism, 4 total) with at least 5 minutes, but no $>10$ minutes, between each measurement. The variance of repeated measurements with each prism from the averaged mean $( \pm S D)$ was examined. Also linear correlation coefficients were calculated for each of the independent variable corneal error parameters examining the CATS-GAT difference across all subjects. Finally, a multiple regression analysis was calculated to examine the effect of multiple independent error parameter variables $($ Dof $=3,95 \% \mathrm{CI})$.

\section{Results}

IOP measurements with the CATS prism and the GAT tonometer prism were completed on 109 eyes of 55 patients. The study's average subject age was $49 \pm 15$ years with 32 females and 23 males.

The difference in CATS and GAT prisms was measured and correlated to CCT. The subject's average CCT was $555 \pm 45 \mu \mathrm{m}$ which is comparable to a similar study at $556 \pm 40 \mu \mathrm{m} .{ }^{14}$ The results shown in Figure 3 confirm the negative GAT correction slope of $-0.024 \mathrm{mmHg} / \mu \mathrm{m}$ of CCT predicted by the design theory, and the difference in CATS and GAT measurements is $\sim 0$ at an average corneal thickness $(556 \mu \mathrm{m})$. The mean IOP with the CATS prism was $17.8 \mathrm{mmHg}$ compared to $17.5 \mathrm{mmHg}$ with the Goldmann. Discarded and repeated measurements out of range occurred twice with the CATS and zero times with the GAT. The CATS prism reduced the IOP error due to CCT by a maximum of $\pm 3 \mathrm{mmHg}$ over the GAT prism, which compares to the published error on GAT prism at $\pm 6 \mathrm{mmHg}$ over this same range of CCT values. ${ }^{6}$ The correlation coefficient associated with CCT error was moderate at $0.43(P=0.02)$, indicating good correlation between the difference in IOP between the CATS and GAT prisms over the range of corresponding CCT values.

The IOP difference in CATS and GAT prisms was measured and correlated to CRF as measured by the ORA. The results shown in Figure 4 also confirm the expected slope of $-0.39 \mathrm{mmHg} / \mathrm{units} \mathrm{CRF}$ predicted by the design theory and the difference in CATS and GAT measurements is $\sim 0$ at an average corneal rigidity. The study CRF measured an average of $9.8 \pm 2.6$. The CATS prism reduces the IOP error

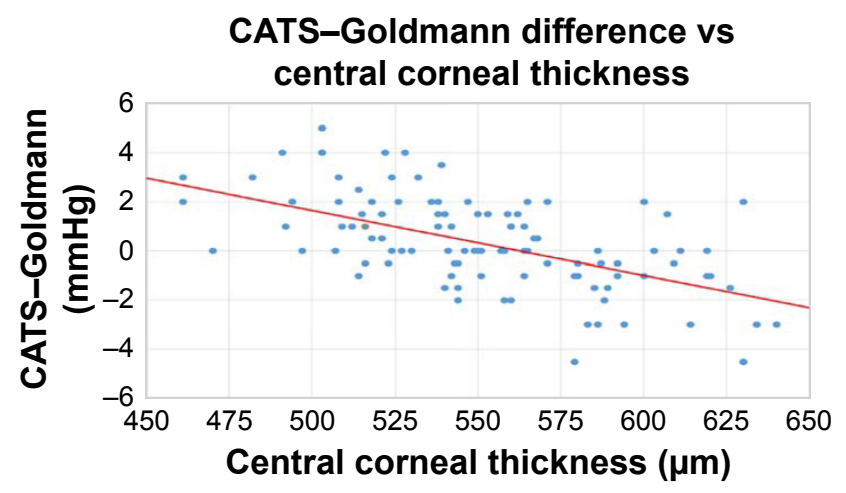

Figure 3 CATS minus GAT IOP difference correlated to CCT.

Abbreviations: CATS, correcting applanation tonometry surface; CCT, central corneal thickness; GAT, Goldmann applanation tonometry; IOP, intraocular pressure. 


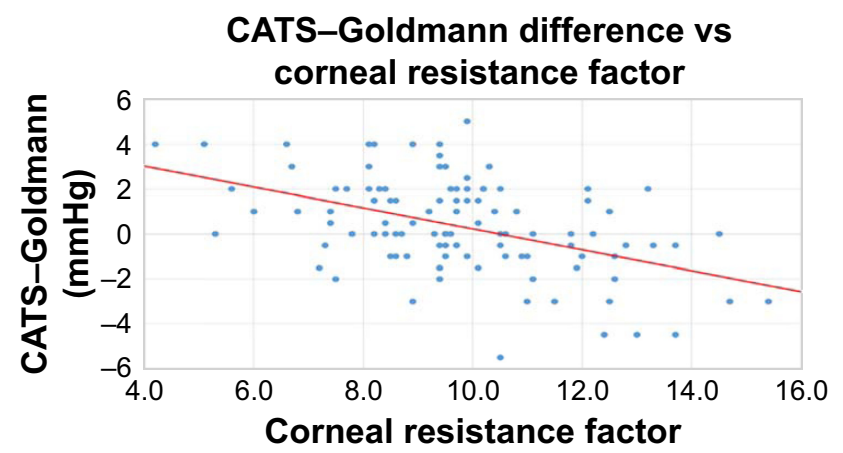

Figure 4 CATS minus GAT IOP difference correlated to CRF.

Abbreviations: CATS, correcting applanation tonometry surface; CRF, corneal resistance factor; GAT, Goldmann applanation tonometry; IOP, intraocular pressure.

due to CRF by an additional $\pm 3 \mathrm{mmHg}$ over the GAT prism at the measured CRF extremes of 4.2 and 15.6. This result compares favorably with the published error using GAT at $\sim \pm 6 \mathrm{mmHg}$ over a measured range of corneal modulus of elasticity values. ${ }^{6}$ The CATS-GAT difference correlation coefficient associated with the CRF error was moderate at $0.27(P=0.19)$.

The difference in CATS and GAT prisms was measured and correlated to corneal curvature. The results shown in Figure 5 confirm the design theory and the zero difference in CATS and GAT measurements at an average measured corneal curvature. The average corneal curvature was $43.6 \pm 1.6$ diopters. The CATS prism reduces the IOP error due to corneal curvature by $\pm 2 \mathrm{mmHg}$ over the GAT prism with a correction slope of $-0.60 \mathrm{mmHg} /$ diopter, which completely corrects the published GAT error over the range of corneal curvature values (40.0-47.0 diopters). ${ }^{6}$ The correlation coefficient associated with corneal curvature error was low at $0.20(P=0.20)$.

The results shown in Figure 6 confirm that the average measurement does not deviate significantly from a 1:1 IOP correlation with a high coefficient of 0.78 . The average

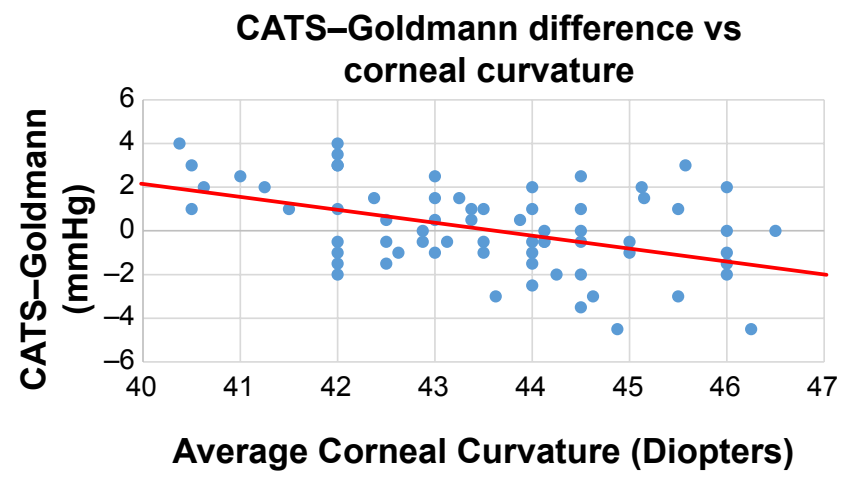

Figure 5 CATS minus GAT, IOP difference correlated to corneal curvature. Abbreviations: CATS, correcting applanation tonometry surface; GAT, Goldmann applanation tonometry; IOP, intraocular pressure.

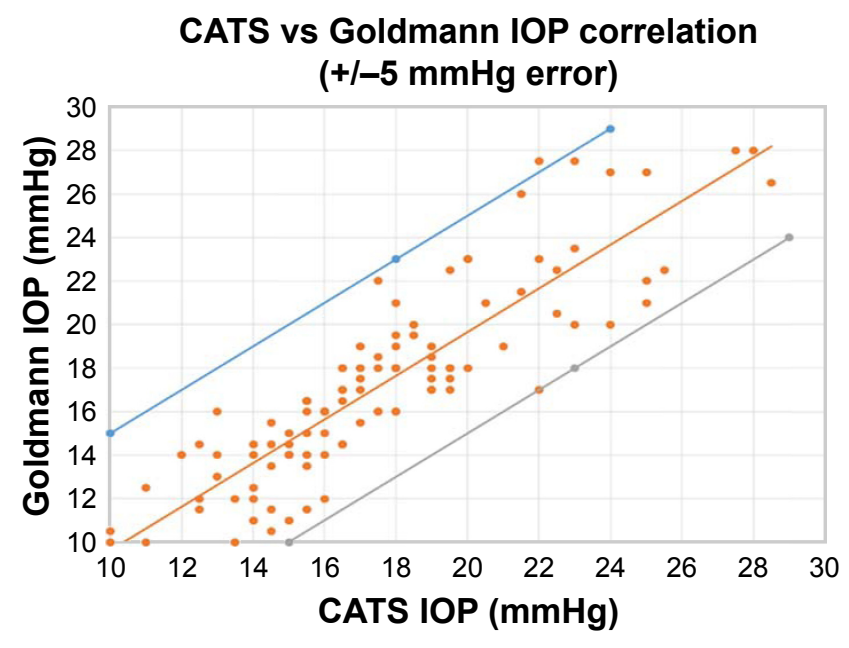

Figure 6 CATS versus GAT, IOP correlation.

Abbreviations: CATS, correcting applanation tonometry surface; GAT, Goldmann applanation tonometry; IOP, intraocular pressure.

difference between the GAT and CATS prisms is negligible throughout a pressure range of $10-28 \mathrm{mmHg}$. A multiple regression analysis (Table 1) found the three corneal biomechanical errors to be correlated with a moderate coefficient of $R^{2}=0.43$, which is similar to other studies examining IOP correlations. ${ }^{19-21}$ The probabilities of null significance with each of the independent variables in the multiple regression are listed in Table 1. A post hoc power calculation of the 109 patients was completed and found to be $98.3 \%$ (alpha $=0.05)$. Since the Tonometer testing ANSI standard 80.10-2014, Annex B, Section B.5.5 allows for both eyes to be utilized without a requirement to independently account for statistical bias in proving tonometers to both the US Food and Drug Administration and European Union for premarket approval, we followed their recommendations. However, even if we consider zero independence between bilateral eye measurements with 55 patients our post hoc power calculation remains high at $82.9 \%$. The variance in repeated IOP measurements indicated a minimal variance of 0.27 (standard deviation $=0.52 \mathrm{mmHg}$ ) with the CATS prism and 0.19 (standard deviation $=0.44 \mathrm{mmHg}$ ) with the GAT prism.

A Bland-Altman analysis is shown for comparison in Figure 7. The mean difference is $0.352 \mathrm{mmHg} \pm 4.11 \mathrm{mmHg}$ $( \pm 1.96 \mathrm{SD})$. There is moderate difference in IOP measurements between the CATS and GAT on the Bland-Altman which confirms the purpose of the study.

\section{Conclusion}

The clinical study shows a significant reduction in CATS prism sensitivity to recognized corneal biomechanical errors in GAT IOP measurement. The results verify the previously published mathematical modeling and the expected slope 
Table I CATS versus GAT statistical analysis

\begin{tabular}{lllll}
\hline Parameter & $\begin{array}{l}\text { GAT correction } \\
\text { slope }\end{array}$ & $\begin{array}{l}\text { Correlation } \\
\text { coefficient }\end{array}$ & $\begin{array}{l}\text { Multiple regression } \\
\text { P-value }\end{array}$ & $\begin{array}{l}\text { Measurement } \\
\text { variance }\end{array}$ \\
\hline Corneal thickness & $-0.024 \mathrm{mmHg} / \mu \mathrm{CCT}$ & 0.43 & 0.02 & - \\
Corneal curvature & $-0.06 \mathrm{mmHg} / \mathrm{Diopter}$ & 0.20 & 0.19 & - \\
Corneal resistance & $-0.39 \mathrm{mmHg} / \mathrm{CRF}$ unit & 0.27 & 0.2 & - \\
Combined multiple regression & - & 0.43 & $\mathrm{~F}<0.01$ & - \\
CATS repeatability & Mean IOP $=17.5 \mathrm{mmHg}$ & - & - & 0.27 \\
GAT repeatability & Mean IOP $=17.8 \mathrm{mmHg}$ & - & - & 0.19 \\
\hline
\end{tabular}

Abbreviations: CATS, correcting applanation tonometry surface; GAT, Goldmann applanation tonometry; IOP, intraocular pressure.

in the difference between CATS and GAT measurements when correlated to each of the error parameters of corneal thickness, corneal rigidity, and corneal curvature. ${ }^{18}$ Only the corneal tear film was not clinically examined which is better suited to a cadaver eye study with static measurement conditions. Low correlation coefficients as seen with the corneal curvature and corneal rigidity correlations are common in clinical IOP studies due to the multiple variables in measurement error. ${ }^{19-21}$ The assumption is that the biomechanical error relationships are linear when in fact there is evidence that they may be nonlinear which may add to a lower correlation. ${ }^{18}$ Correlation coefficients $>0.40$ seen with the CCT, IOP, and multiple regression analysis statistically indicate that the CATS prism is significantly less sensitive to corneal biomechanical errors than GAT IOP measurement.

The results confirm the CATS prism function including the force to pressure conversion supplied by the GAT or Perkins armature remains unchanged. This finding is supported by the zero IOP measurement difference between the CATS and GAT prisms under study averaged error parameters of CCT, CRF, and corneal curvature. Also the direct comparison of IOP measurements between the two prisms averaged over all IOP's indicated a 1:1 correlation further supporting the lack of difference between the two prisms. The previously published study included cadaver eyes

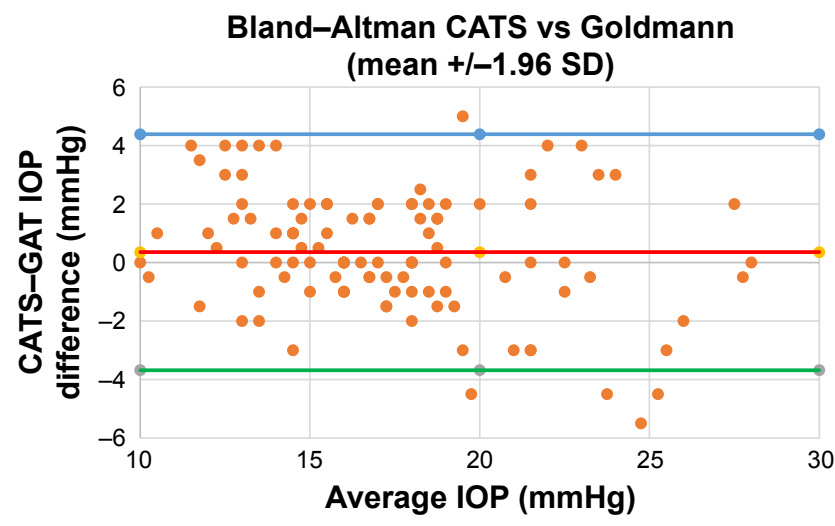

Figure 7 Bland-Altman analysis CATS versus GAT.

Abbreviations: CATS, correcting applanation tonometry surface; GAT, Goldmann applanation tonometry; IOP, intraocular pressure; SD, standard deviation. which also indicated negligible difference between the two prisms when comparing to intracameral transducer measured IOP ${ }^{18}$ Although the average bias is negligible, $\sim 50 \%$ of the population has corneal biomechanical variability which adds significant error to the GAT IOP measurement. ${ }^{6-8}$

The combined error in IOP measurement in published data can total $\pm 15- \pm 19 \mathrm{mmHg}$ for patients at the corneal biomechanical extremes. ${ }^{6}$ The most common recognized error results from individual variations in $\mathrm{CCT}$ at $\pm 7 \mathrm{mmHg}$, which is only a portion of the total potential error and may render CCT correction alone clinically inaccurate. ${ }^{16}$ If only CCT GAT error correction is considered in a standard distribution of varying thicknesses (CCT), then the percentage of the population with CCT errors $> \pm 2 \mathrm{mmHg}$ can be determined. Using the study's CCT population distribution, the percentage of people in which the GAT IOP error is $> \pm 2 \mathrm{mmHg}$ translates to $46 \%$ of all patients from CCT error alone. Using the CATS tonometer prism and the predicted decrease in CCT sensitivity demonstrated by the study, the number of patients with an error $> \pm 2 \mathrm{mmHg}$ is reduced to $3 \%$. The CATS tonometer prism may negate the need for pachymetry measurement with CCT correction, and it simultaneously corrects for other potentially more significant errors as well. The study compared the relative pressure difference between the two prisms and compared it to the expected difference for a given error parameter. Future studies will compare both prisms directly to an intracameral transducer pressure.

Applanation tonometry prisms require a centered cornea on the prism face to accurately measure IOP. The GAT prism will measure applanated mires imaged through the prism anywhere on the flat prism face, but centration is required with the GAT for accurate measurement. The CATS tonometer prism's concave-convex surface does not allow the mires to intersect unless the prism is centered on the cornea. Clinically, within seconds of the first use, the prism was easily centered by all of the investigators during the study and all measurements were serially repeatable demonstrating an equally low repeat measurement variance with both the CATS and GAT prisms. Measurement variability assessment was limited due 
to the number of possible repeat measurements on a given subject in a single setting and the associated corneal toxicity. A cadaver eye study would better assess intraoperator and interoperator measurement variability.

Clinicians today almost universally have the capability to measure IOP with a GAT, and a majority consider it the most accurate measurement of IOP in a normal person. GAT errors are well known to most clinicians and current clinical practice does not correct for most corneal biomechanical errors. However, the CATS tonometer demonstrates the capacity to correct for these inaccuracies and can provide a single error-corrected measurement without additional measurements, calculations, or interpretation error.

\section{Acknowledgments}

This study was supported in part by NIH SBIR Grant R43 EY026821-01 and Arizona Eye Consultants, Tucson, AZ with extensive facilities use. The study was approved and reviewed by Chesapeake IRB. No patient identifiable information is presented. Data used in the study analysis will be made publicly available upon acceptance of the manuscript.

\section{Author contributions}

SM, GL, WD, JL, and CK contributed to the design, collection, and analysis of the study. SM, JS, and ETE contributed to the design, authorship, and revision of the article. All authors contributed toward data analysis, drafting and revising the paper and agree to be accountable for all aspects of the work.

\section{Disclosure}

Sean McCafferty and Jim Schwiegerling have a vested interest in Intuor Technologies which owns the technology being tested in this manuscript. The other authors report no conflicts of interest in this work.

\section{References}

1. Bureau of Labor Statistics. Occupational statistics, 29-1041 Optometrists. 2014.

2. Holden B, Resnikoff S. The role of optometry in vision 2020. Community Eye Health. 2002;15(43):33-36.

3. Quigley H, Broman T. The number of people with glaucoma worldwide in 2010 and 2020. Br J Ophthalmol. 2006;90(3):262-267.

Clinical Ophthalmology

\section{Publish your work in this journal}

Clinical Ophthalmology is an international, peer-reviewed journal covering all subspecialties within ophthalmology. Key topics include: Optometry; Visual science; Pharmacology and drug therapy in eye diseases; Basic Sciences; Primary and Secondary eye care; Patient Safety and Quality of Care Improvements. This journal is indexed on Submit your manuscript here: http://www.dovepress.com/clinical-ophthalmology-journal
4. Susanna JR, De Moraes CG, Cioffi GA, Ritch R. Why do people (still) go blind from glaucoma? Trans Vis Sci Tech. 2015;4(2):1-10.

5. Goldmann H, Schmidt T. Uber Applanationstonometrie. [About Applanation Tonometry...... and other alien spacecraft]. Ophthalmologica. 1957;134(4):221-242. German.

6. Liu J, Roberts C. Influence of cornea biomechanical properties on intraocular pressure measurement: quantitative analysis. J Cataract Refract Surg. 2005;31(1):146-155.

7. Kotecha A, Elsheikh A, Roberts C, Haogang Z, Garway-Heath D. Corneal thickness- and Age Related Biomechanical Properties of the Cornea Measured with the Ocular Response Analyzer. Invest Opthalmol Vis Sci. 2006;47(12):5337-5347.

8. Whitacre M, Stein R. Sources of error with use of Goldmann-type tonometers. Survey Ophthalmol. 2002;38(1):1-30.

9. Neuburger M, Maier P, Böhringer D, Reinhard T, F Jordan J. The impact of corneal edema on intraocular pressure measurements using Goldmann applanation tonometry, Tono-Pen XL, iCare, and ORA: an in vitro model. J Glaucoma. 2013;22(7):584-590.

10. Orssengo G, Pye D. Determination of the true intraocular pressure and modulus of elasticity of the human cornea in vivo. Bull Math Biol. 1999;61:551-572.

11. McCafferty SJ, Schwiegerling JT, Enikov ET. Thermal load from a CO2 laser radiant energy source induces changes in corneal surface asphericity and roughness. Invest Opthalmol Vis Sci. 2012;53:4279-4288.

12. Tselishchev Y, Val'tsifer V. Influence of the type of contact between particles joined by a liquid bridge on the capillary cohesive forces. Colloid J. 2003;65(3):385-389.

13. Orr F, Scriven L, Rivas A. Pendular rings between solids: meniscus properties and capillary force. J Fluid Mech. 1975;67:723-742.

14. Kass M, Heuer D, Higginbotham E, et al. The Ocular Hypertension Treatment Study: a randomized trial determines that topical ocular hypotensive medication delays or prevents the onset of primary openangle glaucoma. Arch Ophthalmol. 2002;120(6):701-713.

15. McCafferty SJ, Schwiegerling JT, Enikov ET. Corneal surface asphericity, roughness, and transverse contraction after uniform scanning excimer laser ablation. Invest Opthalmol Vis Sci. 2012;53(3):1296-1305.

16. Brandt JD, Gordon MO, Gao F Beiser JA, Phillip J. Adjusting intraocular pressure for central corneal thickness does not improve prediction models for primary open-angle glaucoma. Ophthalmology. 2012;119(3):437-442.

17. Elsheikh A, Alhasso D, Gunvant P, Garway-Heath D. Multiparameter correction equation for Goldmann applanation tonometry. Optom Vis Sci. 2011;88(1):E102-E112.

18. McCafferty S, Lim G, Duncan W, Enikov E, Schwiegerling J. Goldmann tonometer prism with an optimized error correcting applanation surface. Transl Vis Sci Technol. 2016;5(5):1-5.

19. Schneider E, Grehn F. Intraocular pressure measurement: comparison of dynamic contour tonometry and Goldmann applanation tonometry. J Glaucoma. 2006;15(1):2-6.

20. Kniestedt C, Nee M, Stamper R. Dynamic contour tonometry: a comparative study on human cadaver eyes. Arch Ophthalmol. 2004;122(9):1287-1293.

21. A-Yong Y, Su-Fang D, Yun-E Z, et al. Correlation between corneal biomechanical properties, applanation tonometry and direct intracameral tonometry. Br J Ophthalmol. 2012;96(5):640-644.

\section{Dovepress}

PubMed Central and CAS, and is the official journal of The Society of Clinical Ophthalmology (SCO). The manuscript management system is completely online and includes a very quick and fair peer-review system, which is all easy to use. Visit http://www.dovepress.com/ testimonials.php to read real quotes from published authors. 